\title{
Editorial for EAIT issue 4, 2021
}

\author{
Arthur Tatnall ${ }^{1}$
}

Accepted: 8 July 2021 / Published online: 13 July 2021

(c) The Author(s), under exclusive licence to Springer Science+Business Media, LLC, part of Springer Nature 2021

Education and Information Technologies (EAIT) is a research journal that covers the complex relationships between Information and Communication Technologies and Education. EAIT is the official journal of the Technical Committee on Education (TC3) of the International Federation for Information Processing (IFIP).

Articles submitted to the journal relate to all levels: the micro level of specific applications or instances of use in classrooms to macro concerns of national policies and major projects; from classes of pre-schoolers to adults in tertiary institutions; from teachers and administrators; to researchers and designers; from institutions to open, distance and lifelong learning. The journal's breadth of coverage allows EAIT to examine fundamental issues at all levels, discuss specific instances and cases, draw inference and probe theory. The journal is embedded in the research and practice of professionals and has been accepted into the Social Science Citation Index (SSCI) in the category 'Education \& Educational Research'.

The first article in this issue: Application of virtual reality for infrastructure management education in civil engineering describes research by Farrukh Arif (NED University of Engineering and Technology, Karachi, Pakistan). The author points out that cultivation of student understanding in civil engineering requires them to conceive things in a manner that allows for a comprehensive grasp of practical aspects in alignment with theoretical principles. Virtual Reality (VR) is technology intervention that provides real-world like sensations to its users within a simulated setting, enabling them to safely entertain, learn, experiment, and feel. This paper presents a study on the application and assessment of using virtual reality for infrastructure management education in civil engineering.

Azza Alawadhi (Higher Colleges of Technology, Ras Al Khaimah Womens College, United Arab Emirates) and Emad A. S. Abu-Ayyash (The British University in Dubai, United Arab Emirates) next offer: Students' perceptions of Kahoot!: An exploratory mixed-method study in EFL undergraduate classrooms in the UAE. Game-based student response systems (GSRS) such as Socrative, Quizlet, and Kahoot! have become a popular tool to increase motivation, enhance classroom

Arthur Tatnall

Arthur.Tatnall@vu.edu.au

$1 \quad$ Victoria University, Melbourne, Australia 
engagement, and facilitate collaborative learning, but little research has attempted to understand student perceptions using these platforms for language learning across higher education in the Middle East. The purpose of this exploratory mixed methods research was to examine undergraduate student perceptions of Kahoot!, a gamebased interactive platform in an English language course at a federal higher education institution in the UAE.

Effects of technology-enhanced constructivist learning on science achievement of students with different cognitive styles comes from: Esra Açıkül Fırat (Adiyaman University, Turkey), Mustafa Serdar Köksal (Hacettepe University, Ankara, Turkey) and Alaattin Bahşi (Ministry of National Education, Bingöl, Turkey). The purpose of this study was to examine the effect of technology-enhanced constructivist learning on science achievement of seventh-grade students with different cognitive styles. Cognitive styles of the students are examined within the frame of Witkin et al. (1977) in terms of field dependent and field independent cognitive styles.

K. R. Pillai and Pallavi Upadhyaya (Manipal Academy of Higher Education, India), Ashish Viswanath Prakash (Indian Institute of Technology, West Bengal, India), Badrinarayan Srirangam Ramaprasad (Justice K. S. Hedge Institute of Management, NITTE, Mangalore, India), H. V. Mukesh and Yogesh Pai (Manipal Academy of Higher Education, India) then write on: End-user satisfaction of technology-enabled assessment in higher education: A coping theory perspective. Their study examines students' coping process of a forced technological intervention in academic outcome assessment in a higher education setting. This is an empirical study grounded in the Coping Model of User Adaptation (CMUA). They found that problem-focused coping has a significant influence on end-user satisfaction, but that emotion-focused coping is insignificant among the students.

Web 2.0 Personal Learning Environments (PLEs) have been proposed as a new method to promote the use of Web 2.0 tools for learner-centred personal learning say Jieun Lim (Daegu National University of Education, Republic of Korea) and Timothy J. Newby (Purdue University, West Lafayette, USA) in their paper: Preservice teachers' attitudes toward Web 2.0 personal learning environments (PLEs): Considering the impact of self-regulation and digital literacy. This study investigates the effects of self-regulating capabilities (such as metacognition and time management), tool literacy, and information literacy on preservice teachers' attitudes toward using Web 2.0 for constructing their PLEs.

Gonçalo Tomás and Miguel Mira da Silva (Universidade de Lisboa, Lisbon, Portugal) and José Bidarra (Universidade Aberta, Lisbon, Portugal) then offer: Supervision of master theses based on Scrum: A case study. They point out that the success rate of a master program on Information Systems and Computer Engineering in a Portuguese university was very unsatisfactory, showing that less than half of the students complete the assigned work in their first term. However, the success rate of a group of students that were supervised based on the Scrum framework was much higher. In this study the authors assess the current situation and identify the benefits of using Scrum to manage master's theses.

The effect of flipped learning on EFL students' writing performance, autonomy, and motivation by Alaá Ismael Challob (University Of Anbar, Ramadi, Iraq) notes that though flipped learning has positively impacted teaching English writing, 
its usefulness in developing students' English writing performance, autonomy, and motivation is still unclear. The reported study addressed the factors available in the flipped learning English writing environment that contribute to this effect, finding that this learning environment has an impact on promoting students' English writing performance, autonomy, and motivation.

The next study: Integration of information and communication technology in teaching: Initial perspectives of senior high school teachers in Ghana examined the Integration of ICT in teaching, focusing on the Kumasi Girls Senior High School in Ghana. It was offered by Valentina Arkorful and Kwaku Anhwere Barfi (University of Cape Coast, Ghana) and Isaac Kwame Aboagye (Kumasi Girls Senior High School, Ghana). The objectives were to examine the extent to which the attitude of teachers influences the integration of ICT in teaching, and to examine the gender differences in the integration of ICT in Senior High Schools.

Issues in learning management systems implementation: A comparison of research perspectives between Australia and China by Darren Turnbull, Ritesh Chugh and Jo Luck (Central Queensland University, North Rockhampton, Australia) points out that Australian post-secondary institutions have embraced the incorporation of Learning Management Systems (LMSs) into traditional classroom-based instruction and distance learning models. Given that an increasing number of Chinese students are studying in Australia and that their experience of using LMSs in China may be very different from that in Australia, it is important to explore the issues in LMS implementation in the two countries. This literature review compares the use of LMSs in China and Australia with the aim of identifying some of the issues impacting their integration into the course offerings of post-secondary institutions in both countries.

Samantha Leonard, Michael J. Stroud and Raymond J. Shaw (Merrimack College, North Andover, USA) next present: Highlighting and taking notes are equally ineffective when Reading paper or eText. The reported study examined whether there is a difference in comprehension when reading from computer-based text versus reading from paper-based text and whether there is any benefit from the ability to take notes and highlight text while reading. The results revealed no significant difference in reading times and comprehension scores across conditions and no significant difference in highlighting and note-taking across text formats.

Implications of the Delphi method in the evaluation of sustainability open education resource repositories by Xiaochen Wang, Tingting Chen and Yihan Zhang (Capital Normal University, Beijing, China) and Harrison Hao Yang (State University of New York at Oswego, USA) aimed to break through the original 'fixed', highly structured evaluation system and develop an open and flexible " $1+\mathrm{X}$ " evaluation framework of open educational resources (OER) by using the modified Delphi method. The framework of this study provides a basis for the evaluation and optimisation of existing OER repositories and also serves as functional scaffolding for the construction of new repositories.

University students have to master new twenty-first century competences to be able to completely form part of Knowledge Society, and Gonzalo Almerich, Jesús Suárez-Rodríguez, Isabel Díaz-García and Natividad Orellana (University of Valencia, Spain) discuss this in: The influence of using ICT in high-skills competences 
and ICT competences. A structural model. These include high-skill competences - higher-order thinking capacities and teamwork competences, and ICT competences - technological, pedagogical and ethical, as well as using technological resources. Their study aimed to determine the influence of ICT use - personal use, academic use at home and academic use in the classroom, on the relational asymmetric structure of ICT competences on high-skill competences.

Mustafa Can Demirkiran (Hatay Bahçeşehir College Antakya, Turkey) and Fatma Tansu Hocanin (Cyprus International University Mersin, Nicosia, Cyprus) next write on: An investigation on primary school students' dispositions towards programming with game-based learning. Today, new technological teaching aids are used to support learning and to increase motivation and adaptation of students. Game-based Learning (GBL) is one such aid. In computer science courses, the concept of programming can be confusing and difficult to understand by some students and this study investigated the disposition of $5^{\text {th }}$ grade primary school students on programming through a digital game-play.

An assessment of the interplay between literacy and digital Technology in Higher Education relates a study by Shahrokh Nikou (Åbo Akademi University, Turku, Finland and Stockholm University, Sweden) and Milla Aavakare (Åbo Akademi University, Turku, Finland). Digital technologies fundamentally transform teaching and learning in higher education environments, with the pace of technological change exacerbating the challenge, and this research developed a conceptual model and employed Structural Equation Modelling using Partial Least Squares to examine the impact of information and digital literacy on Finnish university staff and students' intention to use digital technologies.

Sofia Moya and Mar Camacho (Universitat Rovira i Virgili, Carretera de Valls, Spain) then present: Identifying the key success factors for the adoption of mobile learning. They argue that there is evidence that sustainability and efficiency are challenges for mobile learning adoption methods and the objective of their study was to identify, categorize and evaluate the critical factors that shape the adoption and sustainable use of mobile learning. The study investigated the factors that affect mobile learning adoption, how they can be categorised and the prioritization of these categories in Catalonia. Overall, participants prioritised these from the most challenging to the lowest impact as: leadership; personal character, attitudes, and ethics; digital literacy; pedagogical; and technological resources.

The literature addresses the needs in acclimatising dynamic, student-driven instruction to conceive a significantly enhanced online learning environment, say Asma Almusharraf (Al-Imam Mohammad Ibn Saud Islamic University, Riyadh, Saudi Arabia) and Norah Almusharraf (Prince Sultan University, Riyadh, Saudi Arabia) in their article: Socio-interactive practices and personality within an EFL online learning environments. This study examines the relationship between student characteristics - introversion and extraversion, and contribution in the online writing environment - social presence, and their sense of class community in online writing courses.

Exploring the effectiveness of a mobile writing application for supporting handwriting acquisition of students with dysgraphia is a paper by Sinan Hopcan (Istanbul University - Cerrahpasa, Istanbul, Turkey) and S. Tugba Tokel (Middle 
East Technical University, Ankara, Turkey). Handwriting is one of the most important essential skills for both literacy and beyond, but some students have difficulty in writing. Dysgraphia, a disorder of written expression, is a condition where a student's writing skills are below the level expected of their intelligence, age, and education. The main purpose of this study was to examine the effectiveness of a mobile writing application for students with dysgraphia and to determine whether students' writing skills improve after using this application.

Exploring the determinants of students' academic performance at university level: The mediating role of internet usage continuance intention by Mahmoud Maqableh, Mais Jaradat and Ala'a Azzam (The University of Jordan, Amman, Jordan) follows. Their study investigates the impact of integrating essential factors on academic performance in the context of university students, and the influence of continuance intention, satisfaction, information value, and Internet addiction on academic performance. It also investigates the mediating role of continuance intention on the relationship of satisfaction and information value on academic performance among university students.

Kiran Fahd (Victoria University, Melbourne, Australia), Shah J. Miah (University of Newcastle, Australia), Khandakar Ahmed (Victoria University, Melbourne, Australia), Sitalakshmi Venkatraman (Melbourne Polytechnic, Australia) and Yuan Miao (Victoria University, Melbourne, Australia) next present: Integrating design science research and design based research frameworks for developing education support systems. Their paper proposes an integrated research framework that takes advantage of the similarities of design science research (DSR) and design-based research (DBR) for developing a new decision support system (DSS) artefact.

Ariya Pakinee and Kitti Puritat (Chiang Mai University, Thailand) then wrote on: Designing a gamified e-learning environment for teaching undergraduate ERP course based on big five personality traits. They note that the COVID-19 pandemic forced universities to push the use of distance learning, impacting many students. This study presents an applied gamification concept to e-learning focusing on improving engagement of the various types of personalities of undergraduate students in ERP courses. The gamification design was developed by implementing the pros and cons of each game element to compromise the overall performance of students.

Factors affecting ICT integration in TURKISH education: a systematic review comes from Yigit Emrah Turgut (Recep Tayyip Erdogan University, Rize, Turkey) and Alper Aslan (Cemisgezek Vocational High School, Munzur University, Tunceli, Turkey). The meta-synthesis method is used to make in-depth analysis and to interpret qualitative research findings. Through inclusion and exclusion criteria, 60 studies among 907 candidate studies accessed from databases including the Web of Science, Education Resource Information Center (ERIC) and the Turkish Academic Network and Information Center are included in this research.

The expansion of access to devices, as well as the production and publication options in social networks requires a broader definition of the traditional notion of personal digital storytelling write José Luis Rodríguez-Illera (Universitat de Barcelona, Spain), Elena Barberà Gregori (Universitat Oberta de Catalunya, Barcelona, Spain) and Núria Molas-Castells (Universitat de Barcelona, Spain) in: Reasons and 
mediators in the development and communication of personal digital stories. To get a deeper understanding of these practices in groups of adolescents, the objective of this article was to analyse the reasons that direct publications of personal stories and what kind of filters, agents and consequences are articulated in these stories.

Action protocol for the use of robotics in students with Autism Spectrum Disorders: A systematic review was contributed by: G. Lorenzo, A. Lledó, Pérez-Vázquez and A. Lorenzo-Lledó (University of Alicante, Carretera de San Vicent del Raspeig, Spain). Use of ICT has opened new paths in the intervention of people with Autism Spectrum Disorder (ASD), including intervention based on "social robots". This research reviews the state of production of social robotics in the intervention of students with ASD during 2000-2019 to establish an action protocol in the use of this tool through a list of bibliometric indicators.

The effect of technology-supported collaborative problem solving method on students' achievement and engagement is an article by: Erhan Unal (Vocational School of Distance Education, Afyon Kocatepe University, Turkey) and HasanCakir (Gazi University, Ankara, Turkey). A collaborative problem-solving method was used in the experimental groups of Object-Oriented Programming I-II courses participants, one of them being supported with web 2.0 technologies whereas the other was supported with desktop software and face to face communication. The results indicated that a significant difference exists between the experimental groups while the students' levels of collaborative learning engagement in the experimental groups were significantly higher than the students' in the comparison group.

Rishabh Singh and Devansh Timbadia (NMIMS University, Mumbai, India and Cerebranium, Sepapaja, Tallinn, Estonia), Vidhi Kapoor (Cerebranium, Sepapaja, Tallinn, Estonia and NMIMS University, Mumbai, India), Rishabh Reddy and Prathamesh Churi (NMIMS University, Mumbai, India and Cerebranium, Sepapaja, Tallinn, Estonia) then write on: Question paper generation through progressive model and difficulty calculation on the Promexa Mobile Application. Institutions need to use online learning as a powerful tool for conducting exams and assess the students effectively, so it is important to understand each student's capacities and create a different test based on the required difficulty. Students should be graded based on their capabilities. The purpose of this research study was to develop the progressive model with the calibration of difficulty level according to the student capacity.

The purpose of the next reported research: High school students' acceptance and use of mobile technology in learning mathematics was to investigate factors affecting the acceptance and use of mobile technology in learning mathematics based on the Unified Theory of Acceptance and Use of Technology 2 (UTAUT2) model. It was contributed by Kübra Açıkgül and Süleyman Nihat Şad (İnonu University, Malatya, Turkey). Their results revealed both direct and indirect effects of exogenous variables on Behavioural Intention and Use Behaviour in mobile technology acceptance of high school students in learning mathematics.

Fade Shi (Xi' an Polytechnic University, China) next presents: Using technologybased tools in ensuring quality of publishable journal articles: the case of Chinese undergraduate research writing experience. The researcher notes that undergraduate student engagement in research publication facilitates their intellectual 
development and professional social interaction and leads to an increased sense of self efficacy and trust in science and recognition as a researcher. Scientific publication is a pillar which gauges intellectual human capital of countries in the global innovation index. This paper presents the effectiveness of using technology-based tools in ensuring the quality of articles for journal publication.

Determinants predicting undergraduates' intention to adopt e-learning for studying English in Chinese higher education context: A structural equation modelling approach. This work by Chunming Li and Limin He (Zhaoqing University, Guangdong, China) and IpKin Anthony Wong (Sun Yat-Sen University, Guangdong, China) showed that in addition to perceived usefulness and perceived ease of use, the intrinsic motivation factor of perceived enjoyment and extrinsic motivation factor of social influence can also determine students' intention to apply e-learning for studying English.

The voice, text, and the visual as semiotic companions: an analysis of the materiality and meaning potential of multimodal screen feedback. The gap between how learners interpret and act upon feedback has been widely documented in the research literature. What is less certain is the extent to which the modality and materiality of the feedback influence students' and teachers' perceptions. This article, by Clare Tyrer (Lancaster University, UK) explores the semiotic potential of multimodal screen feedback to enhance written feedback.

Ricardo-Adán Salas-Rueda (Universidad Nacional Autónoma de México) next writes on: Use of flipped classroom in the marketing career during the educational process on financial mathematics with the aim of analysing the use of a flipped classroom in considering data science, machine learning (linear regression) and neural network. The results of machine learning indicated that the consultation of English and Spanish videos before the class, the realization of the exercises collaboratively through spreadsheet and dissemination of the answers about the exercises through Google Drive during the class and realisation of the online exams and laboratory practices after the class positively influence the development of mathematical skills about simple interest.

Christos Troussas, Akrivi Krouska and Cleo Sgouropoulou (University of West Attica, Athens, Greece) next discuss their research on: Impact of social networking for advancing learners' knowledge in E-learning environments. They point out that social networking has modernized digital education through the provision of novel functionalities, such as reacting, commenting, motivation or group formation. In the light of the new developments, this paper presents SNAKE (Social Networking for Advancing Knowledge in E-learning environment), which is an e-learning software incorporating social characteristics for the tutoring of computer programming.

This study: An evaluation of accessibility to preschool education institutions using geographic information systems, aimed to evaluate current locations of preschool education institutions and suggest alternative spatial arrangements in the Afyonkarahisar province in Turkey, using heuristic location-allocation modelling approaches to improve overall spatial accessibility. It describes research by: Mustafa Köse and Mehmet Koçyiğit and Cahit Erdem (Afyon Kocatepe University, Turkey) and Idris Mohammed Jega (National Space Research and Development Agency, 
Abuja, Nigeria). The results of the study showed that provision of alternative preschool locations decreased the average travel distance from each residential home to each preschool by over $600 \mathrm{~m}$, thereby improving overall accessibility to preschools.

Using a video annotation tool to enhance student-teachers' reflective practices and communication competence in consultation practices through a collaborative learning community comes from Mabel Mei-Po Shek and Kim-Chau Leung (The Education University of Hong Kong, Tai Po, Hong Kong SAR) and Peter Yee-Lap To (Lingnan University, Hong Kong SAR). They investigated how peer comments made using a video annotation tool (VAT) through an online learning community improved student-teachers' level of reflective thinking and communication competence throughout the simulated, role-play, context of consultation practice. They found that the VAT could enhance student-teachers' learning by reducing communication barriers created by the tendency to avoid direct observations of peers' weaknesses, enhancing reflection-in-action during practice, and contextualizing written comments by referring to specific video segments. However, student-teachers felt that the VAT should also allow users to draw on the video screens.

Informed by the educational conditions shaped by the coronavirus pandemic and an increased reliance upon online learning solutions and technologies, this article examines the role of personality traits and online academic self-efficacy in acceptance, actual use and achievement in Moodle on a socially distanced asynchronous university course in Japan. The role of personality traits and online academic selfefficacy in acceptance, actual use and achievement in Moodle comes from Damian J. Rivers (Future University Hakodate, Japan). The study found that agreeableness and conscientiousness have positive direct effects on online academic self-efficacy in addition to positive indirect effects on the acceptance of Moodle, and this had an indirect effect on course achievement while none of the five-factor model personality traits had an influence on actual Moodle use.

Hanaa Ouda Khadri Ahmed (Ain Shams University, Cairo, Egypt) next argues that drone technology has great potential for enhancing and improving teaching and learning experiences in GeoSTEM Education which involves the integration between Geosciences and STEM domains in an article titled: Towards application of drone- based GeoSTEM education: Teacher Educators readiness (attitudes, competencies, and obstacles). It is argued that building and flying drones can enhance interdisciplinary approach to GeoSTEM Education as this helps students to use concepts and principles of Robotics, Maths, Radio Communication Systems, Computer Programming, Electronics, Chemistry, Physics, Photography, Flight Dynamics, Media, project monitoring and hands-on experiences.

The next paper, by Norliza Kushairi and Aidi Ahmi (Universiti Utara Malaysia, Kedah, Malaysia) examines the current dynamics of flipped classroom studies and proposes a direction for future research for the field. The paper is titled: Flipped classroom in the second decade of the Millenia: a Bibliometrics analysis with Lotka's law. Using a bibliometric approach they observed a sample of 1557 documents from the Scopus database to identify research activity on the flipped classroom. This paper also predicts the future study in the flipped classroom using Lotka's law. They found that the pattern distribution of the author's contribution fits with the law and conclude by suggesting a few potential research directions on the flipped classroom. 
Effectiveness of game development-based learning for acquiring programming skills in lower secondary education in Croatia it was contributed by Martina Holenko Dlab and Natasa Hoic-Bozic (University of Rijeka, Croatia). A European initiative CODING4GIRLS (C4G) promotes the acquisition of programming skills through a game development process with the aim of preparing young learners, especially girls, to enter computer science careers and raising awareness of the relationship between ICT and the real world. Using the C4G game development-based learning methodology, students develop serious games for which they need to learn specific programming concepts. This paper presents the results of a study with a mixed gender group of both boys and girls carried out with the aim of examining the effectiveness of the $\mathrm{C} 4 \mathrm{G}$ development-based learning approach in lower secondary education in Croatia.

Learning style theories have been widely used in adaptive learning systems to enhance learning outcomes says Zhanni Luo (University of Canterbury, Christchurch, New Zealand) in: Using eye-tracking technology to identify learning styles: Behaviour patterns and identification accuracy. This author proposed to test the feasibility of eye-tracking technology in identifying learning styles with everyday materials as well as identification accuracy and selected the FelderSilverman's learning style model (FSLSM) as a framework, enlisted behaviour patterns that can be used to identify the eight learning styles in the FSLSM model. A quasi-experiment was conducted to test whether these behaviour patterns apply to eye movement differences.

Yu-Ju Lin (Purdue University, West Lafayette, USA) and Hung-chun Wang (National Taiwan Normal University, Taipei City, Taiwan) then write on: Using virtual reality to facilitate learners' creative self-efficacy and intrinsic motivation in an EFL classroom. A class of university students in Taiwan participated in a virtual reality (VR) supported creative project with the instructor's guidance step by step. A creative self-efficacy student scale, an intrinsic motivation inventory and an openended survey were given to the students to explore how the project might have influenced their efficacy for creativity and learning of English.

Qi Zhang and Zhonggen Yu (Beijing Language and Culture University, China) then present: A literature review on the influence of Kahoot! On learning outcomes, interaction, and collaboration. They point out that, initially developed in 2012, Kahoot! is a game-based student response system aiming to transform the class into a game show, but that some people have doubts about its effectiveness as an educational game. Based on past studies, this study explored the influence of Kahoot! on learning outcomes and collaboration including curricular interaction and extracurricular collaboration.

Current trends in the digital transformation of higher education institutions in Russia describes research by Alexey Mikheev (MGIMO University, Moscow, Russia), Yana Serkina (Belgorod State National Research University, Russia) and Alexander Vasyaev (Moscow State Law University named after O.E. Kutafin (MGLA), Moscow, Russia). In an attempt to bring together views and experiences of different actors of the educational sector, three individual online surveys were conducted among administrative staff, teachers, and students of several educational institutions. Detailed analysis of survey outcomes indicated positive and negative trends 
in digitalization of educational processes from the perspective of university administrative staff, teachers, and students.

Analysing peer feedback in asynchronous online discussions: A case study by Soon Yen Foo (Malaysian Institute of Teacher Education, Johor, Malaysia) begins by defining Peer Feedback as comments provided by one student to another with the intention to aid their peers' progress in learning. Responses or feedback provided by peers has been recognised as an integral part of the learning process in online learning environments. A case study, conducted among Economics students from an all-girls high school located at the northern part of Malaysia, found that the most common type of feedback provided by students was categorised at a cognitive dimension, with the affective and the metacognitive dimensions following behind.

Nagihan Tanik Önal and Nezih Önal (Nigde Omer Halisdemir University, Turkey) follow with an article on: The effect of augmented reality on the astronomy achievement and interest level of gifted students. This study was conducted in line with an explanatory sequential mixed-methods design and comprised 51 gifted students (26 in the control group and 25 in the experimental group) attending a Science and Arts Centre. In the application process, astronomy teaching activities supported with augmented reality (AR) were used in an experimental group while astronomy teaching activities suggested in the official science curriculum of the middle school $7^{\text {th }}$ grade were used. The data of the study were collected by using an Astronomy Achievement Test and the Scale of Interest in Astronomy as a pre-test and post-test and an AR Interview form administered only to the experimental group students at the end of the application.

The transition through the first year of university study is challenging for many students, and for students from Culturally and Linguistically Diverse (CALD) backgrounds, commencing studies in an English-Medium Instruction (EMI) university program presents specific challenges say Sameera Tahira Ahmed (United Arab Emirates University, Abu Dhabi, UAE) and Thomas Roche (Southern Cross University, Lismore, Australia) in their paper: Making the connection: Examining the relationship between undergraduate students' digital literacy and academic success in an English medium instruction (EMI) university. This paper focuses on the digital literacy practices of undergraduates at a national public university in a Gulf State.

Open educational practices of SWAYAM programme among research scholars comes from K.Sathish Kumar and M. Mahendraprabu (Alagappa University, Karaikudi, India). This study aimed at describing the open educational resources of the state universities of Tamilnadu research scholars on their SWAYAM programme. It also attempts to determine the research scholars' level of open educational practices on their SWAYAM through appropriate methodological and statistical procedures.

The challenges of instructors' and students' attitudes in digital transformation: A case study of Saudi Universities from Abdulaziz Alhubaishy (Saudi Electronic University, Riyadh, Saudi Arabia) and Abdulmajeed Aljuhani (Taibah University, Medina, Saudi Arabia) notes that Digital Transformation (DT) has become the core motivator for almost all organizations worldwide. To cope with the new demands, Higher Education Institutions are also giving due consideration to digitizing their services, including pedagogical services. This paper extracts, synthesizes, 
categorizes, and prioritizes the challenges hindering the success of DT in Saudi universities.

Measuring in-service teacher self-efficacy for teaching computational thinking: development and validation of the T-STEM CT. The authors, Danielle Cadieux Boulden, Arif Rachmatullah, Kevin M. Oliver and Eric Wiebe (North Carolina State University, Raleigh, USA) say that despite a growing recognition that K-12 teachers should be prepared to teach students computational thinking (CT) skills across disciplines, there is a lack of valid instrumentation that measures teachers' efficacy beliefs to do so. Their study addressed this problem by developing and validating an instrument that measures in-service teachers' self-efficacy beliefs for teaching CT.

Julio Cabero-Almenara (University of Seville, Spain), Francisco D. GuillénGámez (University of Cordoba, Spain), Julio Ruiz-Palmero (University of Malaga, Spain) and Antonio Palacios-Rodríguez (University of Seville, Spain) then present: Digital competence of higher education professor according to DigCompEdu. Statistical research methods with ANOVA between fields of knowledge in different age ranges. They say that the tasks of university educators must be linked to the digital demands posed by the more sophisticated professions of the twenty-first century. The objective of their study was to examine and compare the degree of digital competence of Higher Education educators from different fields of knowledge and different age ranges according to the DigCompEdu framework.

Among teacher beliefs, technology acceptance has a crucial role in effective technology integration into teaching, and Melih Derya Gurer (Bolu Abant Izzet Baysal University, Turkey) writes on this in: Examining technology acceptance of pre-service mathematics teachers in Turkey: A structural equation modelling approach. The purpose of this study was to investigate Turkey's pre-service mathematics teachers' intentions to use technology in their future teaching. The Technology Acceptance Model (TAM) was used as a framework and was expanded with different variables, including facilitating conditions, subjective norms, and technology self-efficacy.

Nexus among cyberloafing behaviour, job demands and job resources: A mediated-moderated model by Hamzah Elrehail (Skyline University College, Sharjah, United Arab Emirates), Shafque Ur Rehman (ILMA University, Karachi, Pakistan), Naveed Iqbal Chaudhry (University of the Punjab, Lahore, Pakistan) and Amro Alzghoul (Amman Arab University, Jordan) examines this through the mediating role of job stress and work engagement and the contingent role of employee motivation at universities. Their research model draws on border theory and the JD-R model.

Ahmet Berk Ustun, Fatma Gizem Karaoglan Yilmaz and Ramazan Yilmaz (Bartın University, Turkey) then offer: Investigating the role of accepting learning management system on students' engagement and sense of community in blended learning. This study was conducted in an introductory computer course with students who participated in a Moodle learning community that was constituted for the blended learning course. Three self-report instruments were employed to gather data from these students studying, finding that their LMS acceptance significantly and positively affected their sense of community and engagement in BL. 
The next study: Effects of self-explanation on applying decision rules in an online learning environment by Yahya İltüzer and Yasemin Demiraslan Çevik (Hacettepe University, Ankara, Turkey) aimed to investigate this with worked examples on university students' skills in applying decision rules, on the retention of these skills, and on the cognitive load in an online learning environment. Two online learning environments to teach decision rules were designed based on worked example method and participants were assigned to an experimental group with selfexplanation strategy and a control group without. The results showed that the online learning process based on the worked examples with self-explanation caused a significant change in the learners' skills of applying decision rules.

Factors influencing instructors' intentions to use information technologies in higher education amid the pandemic is by Ferhan Şahin and Ezgi Doğan (Anadolu University, Eskişehir, Turkey), Ulaş İlic (Pamukkale University, Denizli, Turkey) and Yusuf Levent Şahin (Anadolu University, Eskişehir, Turkey). The purpose of this study was to determine the variables that affect the instructors' intentions to use IT by extending TAM and to examine the roles of individual differences (moderators) in the proposed model. The results showed that self-efficacy, perceived enjoyment, compatibility and facilitating conditions affect the intention to use IT. The most influential construct among these was compatibility.

Andrew Chimpololo (University of Malawi) then offers: Disciplinary variations in the diffusion of heutagogical use of mobile technologies among student-teachers. Whilst teacher education in Malawi seems to be afflicted by numerous challenges emanating from financial inadequacy and human resource constraints, widespread ownership of mobile technologies among student-teachers and versatility of these devices offers prospects for an improved academic environment. This study examines disciplinary variations in the diffusion of heutagogical use of mobile technologies among student-teachers at Domasi College of Education in Malawi.

Computer programming education is often delivered using individual learning strategies leaving group learning techniques as an under-researched pedagogy says Manuel B. Garcia (FEU Institute of Technology, Manila, Philippines) in the following article: Cooperative learning in computer programming: A quasi-experimental evaluation of Jigsaw teaching strategy with novice programmers. This pose a research gap since novice programmers tend to form their own group discussions after lecture meetings and laboratory activities, and often rely on peers when a topic or activity is difficult, and so this study evaluates the impact of cooperative learning using jigsaw technique when teaching computer programming to novice programmers.

Developing gamification e-quizzes based on an Android app: the impact of asynchronous form is by Marwa F. Areed, Mohamed A. Amasha and Rania A. Abougalala (Damietta University, Egypt), Salem Alkhalaf (Qassim University, Alrass, Saudi Arabia) and Dalia Khairy (Damietta University, Egypt). Gamification is very important during the pandemic because it helps students to represent their study contents and enrich their experiences of higher education when learning inperson is unavailable during the pandemic. This study presents an Android-based gamification app to evaluate the effect of using gamification and e-quizzes on college students' learning. 
The aim of the next reported study was to increase knowledge of teachers' understanding and experiences of using, or not using, digital tools in Early Childhood Education (ECE). Digitalisation in early childhood education: a domestication theoretical perspective on teachers' experiences is by Sofe Lindeman (Bergsvägen's Preschool, Stenungsund, Sweden), Maria Svensson (Varekil's Preschool, Henån, Sweden) and Ann-Britt Enochsson (Karlstad University, Sweden). The study takes a domestication theoretical approach based on interviews with twelve teachers from nine ECE institutions working with play-based learning with children aged 1-5 years from four different municipalities in Sweden and answers the following research questions: In what ways, and to what extent, do teachers integrate digital tools in their work? What practical, symbolic or cognitive aspects affect their work with digital tools?

Wei Ching Lee (Republic Polytechnic, Singapore), Wei Leng Neo (Singapore Management University, Singapore), Der-Thanq Chen (Nanyang Technological University, Singapore) and Tzu-Bin Lin (National Taiwan Normal University, Taipei, Taiwan) next write on: Fostering changes in teacher attitudes toward the use of computer simulations: Flexibility, pedagogy, usability and needs. They indicate that while benefits of using computer simulations in teaching and learning are well documented, actual classroom usage remains sporadic. In this study, the researchers interviewed secondary school science teachers in Singapore, to qualitatively unpack the nuances of the affective, behavioural and cognitive dimensions of teacher attitudes towards computer simulation.

Toward a model of learning innovation integration: TPACK-SAMR based analysis of the introduction of a digital learning environment in three Russian universities by Elena Drugova and Irina Zhuravleva (Tomsk State University, Tomsk, Russia), Marina Aiusheeva (Banzarov Buryat State University, Ulan-Ude, Russia) and Daria Grits (Skyeng LLC, Moscow, Russia) points out that digitalisation is penetrating higher education in Russia. Their study explores how three Russian universities have been integrating the Skyes digital learning platform to transform educational practices in English as a foreign language (EFL). The article outlines the main characteristics of the platform, recounts the steps in adopting the technology and addresses the emerging changes to learning and teaching.

Leila Fekri Pileh Roud (University of Tehran, Iran) and Sahbi Hidri (University of Tunis, Tunisia) then write on: Toward a sociocultural approach to computerized dynamic assessment of the TOEFL iBT listening comprehension test. This study addressed the impact of computerised dynamic assessment (C-DA) on the TOEFL iBT listening comprehension test administered to Iranian EFL learners who took part in preparation courses on the TOEFL exam in some language centres in Iran. To mediate the test-takers with hints to process the listening questions, a computer software program was developed, and it was meant to produce: actual, mediated, and learning potential scores. Findings of the study indicated that the actual and mediated scores led to significantly different mean scores in various listening ability levels in almost all question types.

In their paper: Responsive student model in an intelligent tutoring system and its evaluation, Hoang Tieu Binh (Hanoi National University of Education, Vietnam), Nguyen Quang Trung (Vietnam Youth Academy, Hanoi, Vietnam) and Bui 
The Duy (VNU University of Engineering and Technology, Hanoi, Vietnam) introduce a new student responsive model to support students who use an Intelligent Tutoring System (ITS) as an E-Learning tool. They propose a weighted-based model to estimate and suggest learning materials for students who are pursuing a computerbased course. They have built a brand new ITS called WinITS with threir proposed responsive student model and deployed it in Hanoi National University of Education-Vietnam (HNUE) in the second semester of the school year 2019-2020 with a computer science course.

An ontology-based hybrid e-learning content recommender system for alleviating the cold-start problem is from Joy Jeevamol and V. G. Renumol (Cochin University of Science and Technology, Kerala, India). They note that an e-learning recommender system (RS) aims to generate personalised recommendations based on learner preferences and goals. The existing RSs in the e-learning domain still exhibit drawbacks due to inability to consider the learner characteristics in the recommendation process. This paper deals with the new user cold-start problem, which is a major drawback in e-learning content RSs.

This issue contains articles from researchers in the following countries: Australia, China, Croatia, Egypt, Estonia, Finland, Ghana, Greece, Hong Kong SAR, India, Iran, Iraq, Japan, Jordan, Malawi, Malaysia, México, New Zealand, Nigeria, Pakistan, Philippines, Portugal, Republic of Korea, Russia, Saudi Arabia, Singapore, Spain, Sweden, Taiwan, Thailand, Tunisia, Turkey, UK, United Arab Emirates, USA and Vietnam.

Dedicated, professional reviewers are an essential part of any research journal and EAIT is fortunate to have some excellent reviewers. We do, however, have a problem. We do not have enough reviewers! In 2020 we had over 1300 submissions to the journal (288 articles were published), and in the first six months of 2021 we have received 1100 submissions. This means a huge effort for our present number of reviewers. We need more people to register as reviewers and we encourage readers to consider registering and inviting their colleagues also to register. To do this, all you need to do is to go to the EAIT Editorial Manager (https://www.editorialm anager.com/eait/default.aspx) and click on Register Now.

Publisher's note Springer Nature remains neutral with regard to jurisdictional claims in published maps and institutional affiliations. 